\title{
Studying the effects of environmental conditions on the quality of quinoa seeds
}

\author{
Sara Granado Rodríguez *, Luis Felipe Pérez-Romero, Javier Matías, Nieves Aparicio, Luis \\ Bolaños and María Reguera
}

\begin{abstract}
Quinoa is an Andean crop which has gained worldwide attention in the last few decades. This is in part due to the high nutritional value of its seeds (that present high protein content, a balanced amino acid composition and no gluten), and because of its remarkable capacity to grow and adapt to a wide range of agroecological conditions. Despite the importance of developing nutritious varieties to contribute to food security, little efforts have been made trying to identify the molecular pathways responsible for changes in the nutritional properties of the seeds under changing environmental conditions. In this study we aim to evaluate the effect that the adaptation to different environmental conditions has on the nutritional profile of quinoa seeds. For this purpose, we analyzed different physiological and nutritional seed's traits in six different quinoa varieties grown in the field during three consecutive years (2017, 2018 and 2019). We found that there were differences between cultivars in most of the nutritional parameters analyzed and that the annuity was a determinant factor in every parameter tested, being 2018 the year with lower yields, germinative rates and antioxidant capacity but with higher seed weights and protein contents.
\end{abstract}

Keywords: quinoa; nutriotional quality; agronomy; food science 